\title{
Interaction of BDNF and COMT Polymorphisms on Paired-Associative Stimulation-Induced Cortical Plasticity
}

\author{
A. Veronica Witte, ${ }^{1,2}$ Julia Kürten, ${ }^{4}$ Stefanie Jansen, ${ }^{4}$ Anja Schirmacher, ${ }^{4}$ Eva Brand, ${ }^{5}$ Jens Sommer, ${ }^{6}$ and Agnes Flöel ${ }^{1,2,3}$ \\ ${ }^{1}$ Department of Neurology, ${ }^{2}$ NeuroCure Cluster of Excellence, and ${ }^{3}$ Center for Stroke Research Berlin, Charité-Universitätsmedizin Berlin, 10117 Berlin, \\ Germany, ${ }^{4}$ Department of Neurology, and ${ }^{5}$ Internal Medicine D, Department of Nephrology, Hypertension, and Rheumatology, University of Münster, \\ 48149 Münster, Germany, and 'Department of Psychiatry and Psychotherapy, Philipps-University Marburg, 35039 Marburg, Germany
}

The common single-nucleotide polymorphism (SNP) brain-derived neurotrophic factor (BDNF) valine-to-methionine substitution at codon 66 (Val66Met) has been associated with differences in memory functions and cortical plasticity following brain stimulation. Other studies could not confirm these results, though, and potential interactions of BDNF carrier status with other learning-relevant SNPs are largely unknown. The present study aimed to evaluate the effects of BDNF Val66Met genotype on paired associative stimulation (PAS)induced motor cortex plasticity, while additionally taking catechol- $O$-methyltransferase (COMT) Val158Met and kidney and brain (KIBRA) rs17070145 carrier status into account. Therefore, a cohort of $2 \times 16$ age- and education-matched healthy young females underwent transcranial magnetic stimulation using an excitatory $\mathrm{PAS}_{25}$ protocol to induce cortical plasticity. Cognitive performance was assessed using implicit grammar- and motor-learning tasks and a detailed neuropsychological test battery. While BDNF carrier status alone did not significantly influence PAS-induced cortical plasticity, we found a significant BDNF $\times$ COMT interaction, showing higher plasticity immediately following the $\mathrm{PAS}_{25}$ protocol for the BDNF Val/Val vs Met genotype in COMT Met homozygotes only $(\mathrm{ANOVA}, p=$ 0.027). A similar advantage for this group was noted for implicit grammar learning (ANOVA, $p=0.021$ ). Accounting for KIBRA rs17070145 did not explain significant variance. Our findings for the first time demonstrate an interaction of BDNF by COMT on human cortical plasticity. Moreover, they show that genotype-related differences in neurophysiology translate into behavioral differences. These findings might contribute to a better understanding of the mechanisms of interindividual differences in cognition.

\section{Introduction}

Common polymorphisms in genes that encode neuromodulatory proteins, including brain-derived neurotrophic factor (BDNF), catechol-O-methyltransferase (COMT), and kidney and brain (KIBRA), may contribute to interindividual differences in cognitive performance (Egan et al., 2001, 2003; Chen et al., 2006; Papassotiropoulos et al., 2006).

One of the most extensively studied polymorphisms, a singlenucleotide polymorphism (SNP) at nucleotide 196 (G/A) in the BDNF gene has been associated with decreased activitydependent secretion of BDNF, due to a valine-to-methionine substitution at codon 66 (Val66Met) (Egan et al., 2003). Thus, Met allele carriers are thought to exhibit lower BDNF levels than $\mathrm{Val} / \mathrm{Val}$ homozygotes, and animal models provided good evidence that lower levels of the neurotrophin BDNF are linked with impairments in hippocampal long-term potentiation (LTP), syn-

\footnotetext{
Received Dec. 2, 2011; revised Jan. 12, 2012; accepted Jan. 19, 2012.

Author contributions: A.F. designed research; J.K., S.J., A.S., and J.S. performed research; A.V.W., J.K., A.S., J.S., and A.F. analyzed data; A.V.W., J.K., and A.F. wrote the paper.

This work was supported by grants from the Deutsche Forschungsgemeinschaft (FI 379-4/2, Fl 379-8/1, and DFG-Exc 257), the Else-Kröner Fresenius Stiftung (2009-141), the Interdisciplinary Center of Clinical Research Münster (Floe3/004/08), and the Bundesministerium für Bildung und Forschung (FKZ 0315673A and 01E00801).

Correspondence should be addressed to Dr. Agnes Flöel, Universitätsmedizin Charité, CCM, Department of Neurology, Charitéplatz 1, 10117 Berlin, Germany. E-mail: agnes.floee@@charite.de.

DOI:10.1523/JNEUROSCI.6010-11.2012

Copyright $\odot 2012$ the authors $\quad 0270-6474 / 12 / 324553-09 \$ 15.00 / 0$
}

aptic integrity, and hippocampus-dependent learning (Patterson et al., 1996; Mizuno et al., 2000; Poo, 2001).

In humans, cortical plasticity has been assessed for BDNF Val66Met in several studies. Using noninvasive brain stimulation protocols, reduced plasticity in BDNF Met allele carriers was seen for most, but not all, protocols and techniques (Kleim et al., 2006) (for review, see Cheeran et al., 2009). Cheeran et al. (2008) reported a trend for lower cortical plasticity, as determined using peripheral electric stimulation and subsequent transcranial magnetic stimulation (TMS) (Stefan et al., 2000), in BDNF Met carriers compared with $\mathrm{Val} / \mathrm{Val}$ homozygotes. Likewise, others reported higher plasticity-induction in Val/Val homozygotes using short-term training and cortical mapping (Kleim et al., 2006; McHughen et al., 2010) or intermittent theta-burst stimulation (iTBS) (Antal et al., 2010). On the other hand, Antal et al. (2010) found larger transcranial direct current stimulation (tDCS)induced modulation of motor cortex excitability in Met carriers. Others failed to detect significant differences between BDNF Val66Met genotype groups for repetitive TMS- or iTBS-induced plasticity (Li Voti et al., 2011).

The above-described results for BDNF Val66Met may in part be explained by study-specific differences in the stimulation protocols or study populations. However, potential gene-gene interactions, e.g., between BDNF Val66Met and COMT Val158Met (Erickson et al., 2008; Nagel et al., 2008), had not been considered (Kleim et al., 2006; Cheeran et al., 2008). Notably, data from studies on cognition suggest that a significant interactive effect 


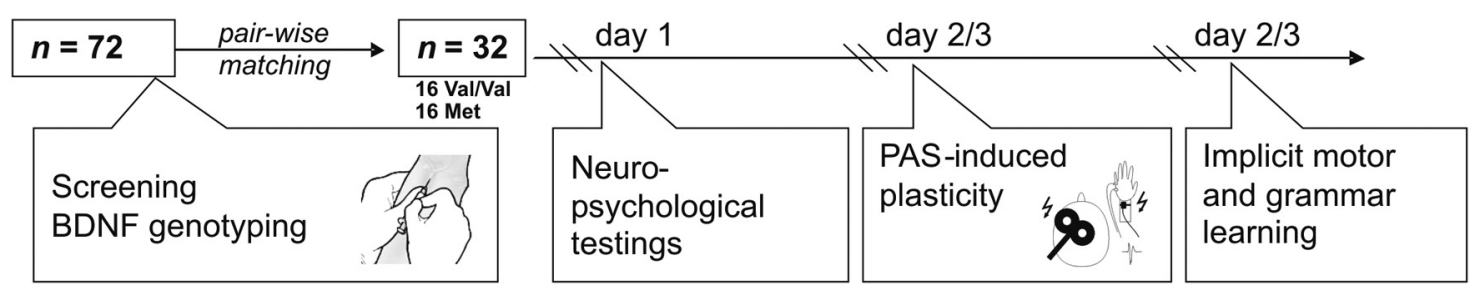

Figure 1. Study flow. At the screening session, subjects underwent medical examination and donated blood samples for genotyping analyses. After identification of 16 female BDNF Met carriers and 16 age- and education-matched BDNF Val/Val carriers, these subjects were tested on cognitive performance using standard neuropsychological tests (day 1; for details, see Materials and Methods). In a randomized order on day 2 or 3, subjects underwent assessment of plasticity of the motor cortex, using a $\mathrm{PAS}_{25}$ protocol (adapted from Stefan et al., 2000). On the other day, implicit motor and grammar learning were assessed using a SRTT and an AGL task.

may exist that may turn a nonsignificant association, for example between BDNF and executive function, into a significant association if the interaction between BDNF and COMT Val158Met is taken into account (Nagel et al., 2008). The COMT Met allele has been linked with less enzymatic activity of COMT and thus higher prefrontal dopamine (Lindenberger et al., 2008). Several studies reported better executive function and memory performance in COMT Met carriers; however, others could not confirm these results (for review, see Witte and Flöel, 2011). For an SNP at intron 9 in the KIBRA gene (rs17070145), both advantages (Papassotiropoulos et al., 2006) and disadvantages have been reported for a C-to-T substitution (for review, see Wersching et al., 2011). The KIBRA protein is discussed to be involved in brain development and memory formation as a postsynaptic scaffold protein (Johannsen et al., 2008; Yoshihama et al., 2009). No study so far assessed the impact of KIBRA rs 17070145 or COMT Val158Met on cortical plasticity using neurophysiological measurements.

Together, the impact of BDNF Val66Met on neuronal plasticity remains controversial, and interactions of BDNF carrier status with other learning-relevant SNPs are not yet understood. Therefore, the present study aimed to determine the effects of Val66Met on LTP-like synaptic plasticity, while additionally accounting for COMT Val158Met and KIBRA rs17070145 carrier status. Moreover, a comprehensive assessment of implicit learning ability and neuropsychological test performance was taken. To avoid confounding by gender (Wersching et al., 2011), a female-only cohort was studied comprising 16 healthy, young Val homozygotes and 16 Met carriers who were matched for age and education.

\section{Materials and Methods}

\section{Subjects}

Seventy-six healthy young volunteers were initially recruited via advertisements in local newspapers and university websites. Peripheral blood samples were collected for genetic analyses if meeting the following inclusion criteria: (1) no history of neurological or psychiatric disorders, (2) no drug abuse or CNS medication, (3) right-handedness ( $>70 \%$ on the Edinburgh Handedness Inventory) (Oldfield, 1971), (4) native German speaker, and (5) normal or corrected-to-normal vision. To avoid confounding by gender (Wersching et al., 2011), only female participants were chosen. Of the initial sample, 16 female BDNF Met allele carriers ( 15 $\mathrm{Val} / \mathrm{Met}, 1 \mathrm{Met} / \mathrm{Met}$; age, $26.9 \pm 8.5$ years, mean $\pm \mathrm{SD}$ ) were identified and pairwise matched for age and years of education to 16 homozygous BDNF Val/Val carriers. These 32 women were invited to participate in three experimental sessions, assessing cortical plasticity as induced by the excitatory paired associative stimulation $\left(\mathrm{PAS}_{25}\right)$ protocol, implicit learning in the motor domain [serial reaction time task (SRTT)] and in the verbal domain [artificial grammar learning (AGL)], as well as neuropsychological performance (Fig. 1, experimental outline). All participants were of Caucasian ethnicity (for demographic details, see Table 1).
Table 1. Demographics of BDNF genotype groups

\begin{tabular}{llll}
\hline & $\begin{array}{l}\text { Met carriers } \\
(n=16)\end{array}$ & $\begin{array}{l}\text { Val/Val carriers } \\
(n=16)\end{array}$ & $p^{*}$ \\
\hline Age (years) & $26.9 \pm 8.5(20-49)$ & $26.5 \pm 8.1(19-49)$ & 0.91 \\
Education (years) & $16.6 \pm 2.2(14-21)$ & $16.2 \pm 2.8(10-21)$ & 0.31 \\
Right-handedness (\%) & $92.5 \pm 9.3(80-100)$ & $95.6 \pm 6.3(80-100)$ & 0.31 \\
BDI & $3.6 \pm 4.8(0-15)$ & $3.9 \pm 4.8(0-18)$ & 0.91 \\
\hline
\end{tabular}

Data expressed as mean $\pm S D$ and [range (minimum to maximum)]. Note that genotype groups did not differ with regard to age, education, handedness, and scores on the Beck's Depression Index (BDI). Handedness score were determined according to the Edinburgh Handedness Inventory. *Paired $t$ test or Wilcoxon rank test, as appropriate.

Table 2. Distribution of BNDF $\times$ COMT and BDNF $\times$ KIBRA genotype

\begin{tabular}{lll}
\hline & BNDF Val66Met & \\
\cline { 2 - 3 } & Met carriers $(n=16)$ & Val/Val carriers $(n=16)$ \\
\hline COMT Val158Met & & \\
Met/Met carriers & 6 & 4 \\
Val carriers & 10 & 12 \\
KIBRA rs17070145 & & 7 \\
T carriers & 7 & 9 \\
C/C carriers & 9 & \\
\hline
\end{tabular}

The study was approved by the local ethics committee of the University of Münster and was conducted in accordance with the Declaration of Helsinki. All participants gave written informed consent.

\section{Genotyping}

DNA was extracted from whole blood using a blood mini-kit (Quiagen). We used GenBank sequences (http://genome.ucsc.edu) to identify the common coding variant in the BDNF gene, a $G \rightarrow$ A polymorphism responsible for the Val66Met change (Sen et al., 2003). Based on a primer database (http://primer3.sourceforge.net/), we designed sequencing primers (BDNF; forward, AGAGTGATGACCATCCTTTTCC; reverse, GCCCGAACTTTCTGGTCCTC) using a Big Dye Terminator Cycle Sequencing Ready Reactio Kit (Applied Biosystems), resulting in a $241 \mathrm{bp}$ PCR product. Amplification reactions were performed using TaqPolymerase (Quiagen), followed by gel electrophoresis of the PCR products. Sequencing was done on a 3730 DNA-Analyzer (Applied Biosystems). For further details, see Sen et al. (2003).

To assess potential interaction effects between BDNF genotype and other learning-relevant polymorphisms, participants were additionally screened for COMT Val158Met and KIBRA rs17070145 genotype status using standard procedures (for details, see Witte et al., 2010; Wersching et al., 2011). Genotyping revealed 25 COMT Met allele carriers (10 Met/ Met, $15 \mathrm{Val} / \mathrm{Met}), 7$ COMT Val/Val carriers, 14 KIBRA rs17070145 $\mathrm{T}$-allele carriers $(\mathrm{T} \rightarrow \mathrm{C}$ allele substitution at intron $9 ; 4 \mathrm{~T} / \mathrm{T}, 10 \mathrm{C} / \mathrm{T})$, and $18 \mathrm{C} / \mathrm{C}$ carriers (for group stratification based on BDNF genotype, see Table 2). Distributions of BDNF and COMT genotypes were in HardyWeinberg equilibrium $\left(\chi^{2}<1.65, p>0.05\right)$.

\section{Neurophysiological measurements}

Determination of baseline stimulation parameters. Single-pulse TMS was performed using a Magstim 200 connected to a figure-eight coil with an outer diameter of $9.5 \mathrm{~cm}$. TMS was delivered to the motor cortex of the 

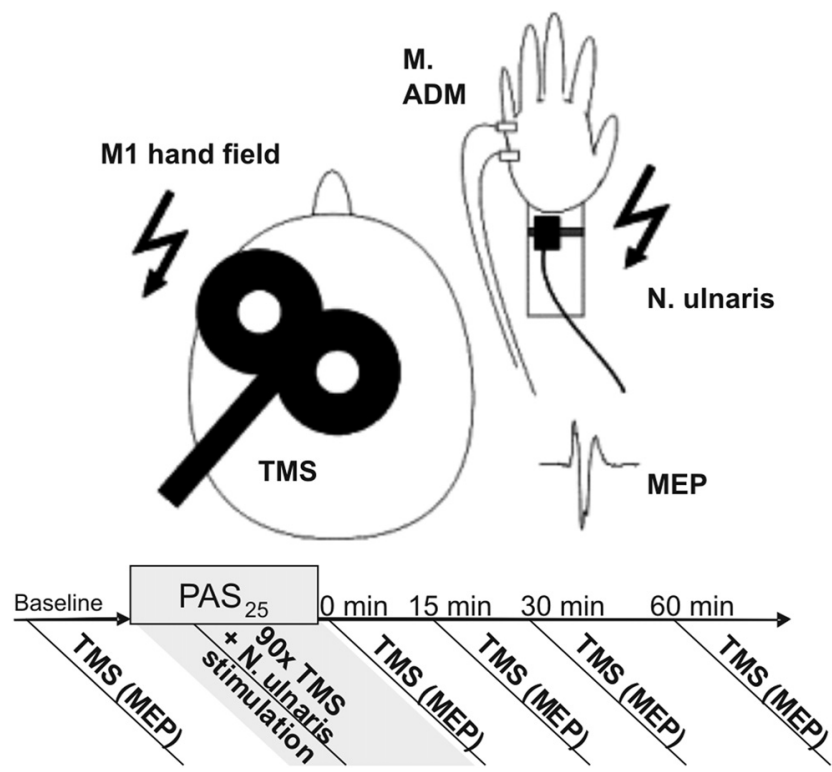

Figure 2. Cortical plasticity assessed by TMS using the excitatory PAS 25 protocol. TMS of the hand field of the contralateral motor cortex (M1) was used to evoke MEPs of the ADM muscle of the right hand. During $\mathrm{PAS}_{25}$, TMS was simultaneously administered with peripheral electrical stimulation of the right $\mathrm{N}$. ulnaris for 90 times (flashes) with an interstimulus interval of $25 \mathrm{~ms}$. Single TMS-induced MEPs were collected at baseline (pre) and at post 0, post 15, post 30, and post 60 after $\mathrm{PAS}_{25}$. An increase in MEP amplitudes after $\mathrm{PAS}_{25}$ indicates induction of synaptic plasticity in M1.

left hemisphere. The optimal coil position ("hot spot") was defined as the location over the motor cortex producing the highest and most stable motor evoked potential (MEP) amplitudes of the abductor digiti minimi (ADM) muscle. During stimulation the coil was held tangentially to the participant's skull at an angle of $45^{\circ}$ to the sagittal plane and with the handle pointing backward (Fig. 2). Resting motor threshold (RMT) was defined as the lowest stimulation intensity that could evoke MEPs of 50 $\mu \mathrm{V}$ in 5 of 10 consecutive pulses. The intensity of stimulation was given as the percentage of maximal stimulator output. MEPs of the ADM were recorded via surface $\mathrm{EMG}$ activity using disposable $\mathrm{Ag}$ - $\mathrm{AgCl}$ electrodes in a belly tendon montage. MEP signals were amplified and bandpass filtered between $100 \mathrm{~Hz}$ and $10 \mathrm{kHz}$ (Portable Keypoint).

PAS-induced cortical plasticity. All subjects underwent the PAS protocol that was similar to the one initially described by Stefan et al. (2000) (see also List et al., 2011). PAS consisted of 90 pairs of electrical stimulation of the ulnar nerve ( $300 \%$ of individual sensory threshold) at the wrist combined with suprathreshold TMS impulse over the hot spot of the $\mathrm{ADM}$ over the contralateral hemisphere at a rate of $0.05 \mathrm{~Hz}$ (total duration of stimulation protocol, $30 \mathrm{~min}$ ). The stimulator output was set to induce MEP values of $0.5-1 \mathrm{mV}$. Interstimulus interval was set to 25 $\mathrm{ms}$, which has been shown to induce LTP-like increase in MEP amplitude (Stefan et al., 2000). MEP amplitude measurements (average of 20 responses) were performed at baseline (pre) and at $0 \mathrm{~min}$ (post 0), $15 \mathrm{~min}$ (post 15), $30 \mathrm{~min}$ (post 30), and $60 \mathrm{~min}$ (post 60) after $\mathrm{PAS}_{25}$ (Fig. 2). As primary outcome measures, MEP amplitudes of each time point were averaged and normalized to the MEP amplitude at baseline for each subject. Additionally, the grand average of adjusted MEPs at post 0 , post 15 , post 30 , and post 60 were calculated, indicating responders (value $>1.0$ ) and nonresponders (value $\leq 1.0$ ) to the PAS $_{25}$ protocol (MullerDahlhaus et al., 2008). To maintain a standardized level of attention during the PAS intervention, subjects were instructed to stay alert, voluntarily relax the ADM of the dominant arm, and count the number of ulnar nerve stimulations. Muscle relaxation was continuously monitored by visual feedback from the surface EMG.

\section{Implicit learning}

Artificial grammar learning. To assess implicit learning in the language domain, we used an AGL task, originally designed by Reber (1967).
Therefore, 200 sequences of the letters $\mathrm{M}, \mathrm{V}, \mathrm{S}, \mathrm{R}$, and X were generated following a finite state grammar by Reber (1967). The Reber grammar defines the possible arrangements of the letters dependent on a grammatical rule [e.g., that the letter S can follow letters M (repeatedly) and V (once), but not X and R; thus, MSVS and MSSVS would be correct, but not MSXS, MSSXS, or MSVSS]. One hundred of the correct letter strings, consisting of 4 to 12 letters, were used as grammatical stimuli in an initial acquisition task. Here, subjects were asked to pay close attention and to retype each string that appeared on a computer screen, without feedback. Subjects were unaware of the underlying concept to ensure implicit learning of the grammar. Subsequently, subjects were told that the strings followed a certain grammatical rule, and asked to discriminate between grammatical and nongrammatical items in a following classification task. As stimuli for this classification phase, another 100 grammatical strings plus 50 nongrammatical strings (created by switching two nonterminal letters) were presented in random order on the screen. Subjects were instructed to respond spontaneously whether they thought the presented stimulus was grammatical or nongrammatical.

Numbers of "hits" and "misses" [for grammatical (G) items], and "false alarms" and "correct rejections" [for nongrammatical (NG) items] of the classification task were assessed. Overall hit rate for G items and overall false alarms for NG items created the "endorsement rate" (percentage of items perceived as grammatical), a frequently used variable in the AGL literature to assess syntactic violation detection (Lieberman et al., 2004; Forkstam et al., 2006; de Vries et al., 2010; Kurten et al., 2010). Additionally, $d$ ' values were calculated, a measure of classification accuracy controlling for response bias (Hochhaus, 1972; Flöel et al., 2009).

Serial reaction time task. To assess implicit learning in the motor domain, we measured performance of an SRTT (Nissen and Bullemer, 1987; Cleeremans and McClelland, 1991; Jiménez et al., 1996), using a modified version with a probabilistic sequence ( $85 \%$ sequential elements plus $15 \%$ random elements). The sequential structure was generated by a finite-state grammar that defined the permissible transitions between successive stimuli. Each stimulus, presented on a computer screen, consisted of an asterisk as substitution of one of five dashes in a row, serving as indicator of the finger 1 (thumb), 2, 3, 4, or 5 (little finger). Subjects had their right hand on a custom-made keypad, which provided a key underlying each fingertip, and were asked to respond to each stimulus by pressing the corresponding key as fast as possible. Subjects had to perform 8 blocks of 250 sequential and random stimuli each. Five hundred milliseconds (ms) after each keypress, the subsequent stimulus appeared, and subjects had a resting interval of 3 min between blocks. No feedback was provided during the task. Reaction times (RTs, in ms) were recorded throughout the experiment and averaged for random and sequential stimuli among blocks. While subjects were familiarized with the task during the first four blocks, mean RT differences between random and sequential stimuli of the last four blocks was taken as outcome measure of procedural motor learning, similar to previous studies (Cleeremans and McClelland, 1991; Rösser et al., 2008).

\section{Neuropychological testing}

We used a comprehensive neuropsychological test battery to assess memory functions, executive functions, and general intellectual abilities (for an overview, see Table 3 ).

Briefly, in the Auditory Verbal Learning Task (German version) (Helmstaedter and Kurthen, 2001), subjects were asked to learn and recall a list of 15 words, with delayed retrieval and recognition trials after 30 min (delayed episodic memory). In subtests of the Wechsler Memory Scale-Revised (Elwood, 1991), subjects had to recall and modify small sequences of digits and blocks, and to learn and recall verbal and visual pairs, with immediate and delayed trials. In the Rey-Osterrieth Complex Figure Test (Meyers et al., 1996), participants were instructed to copy a detailed figure and to recall it after a delay of $30 \mathrm{~min}$. In the Trail Making Test (Spreen and Strauss, 1998) subjects have to join with a pencil on a sheet different digits (part A) or alternating digits and letters (part B) as fast as possible in the right order. In the Regensburger Verbal Fluency Test (German version) (Aschenbrenner et al., 2000), subjects were asked to produce as many words as possible, according to a specific initial letter or a semantic category, or to switch between two letters or two categories. 
Table 3. Neuropsychological testing

\begin{tabular}{ll}
\hline Domain & Test \\
\hline Learning and episodic memory & AVLT, sum of trials 1-5 (learning) \\
& AVLT, trial 6 (recall after distraction) \\
& AVLT, trial 7 (delayed) \\
& AVLT, recognition trial (delayed) \\
& WMS-R, verbal paired associates \\
& WMS-R, visual paired associates \\
Visuospatial construction and memory & RCFT, copy \\
& RCFT, delayed copy \\
Attention and working memory & TMT, Part A TMT, Part B WMS-R, digit span WMS-R, \\
& block span \\
Verbal fluency & RWT, lexical RWT, lexical category change RWT \\
General intellectual abilities & semantic RWT semantic, category change
\end{tabular}

All subjects were tested using German versions of the Auditory Verbal Learning Task (AVLT; Helmstaedter and Kurthen, 2001); Wechsler Memory Scale-Revised (WMS-R; Elwood, 1991); Rey-0sterrieth Complex Figure Test (RCFT; Meyers et al., 1996); Trail Making Test (TMT; Spreen and Strauss, 1998); Regensburger Verbal Fluency Test (RWT; Aschenbrenner et al., 2000); and Hamburg-Wechsler Intelligence Scale for Adults-Revision (HAWIE-R, short form; Tewes, 1991).

Table 4. TMS parameters at baseline dependent on BDNF-genotype

\begin{tabular}{lccl}
\hline & Met carriers & Val/Val carriers & $p^{*}$ \\
\hline RMT $(\%)$ & $39.9 \pm 5$ & $41.1 \pm 8$ & 0.54 \\
Baseline MEP $(\mu \mathrm{V})$ & $775 \pm 363$ & $707.3 \pm 209$ & 0.68 \\
Stimulation intensity $(\%)$ & $49.8 \pm 7$ & $51.9 \pm 9$ & 0.42 \\
Perceptual threshold $(\mathrm{mA})$ & $2.6 \pm 0.9$ & $3.3 \pm 1.1$ & 0.094
\end{tabular}

RMT and stimulation intensity to evoke a $0.5-1 \mathrm{mV}$ MEP are given as percentage of maximum stimulator output. Data are given as mean \pm SD. No significant group differences were found. *Paired $t$ test or Wilcoxon rank test, as appropriate.

The short version of the Hamburg Wechsler Intelligence Scale for Adultsrevised (German version) (Tewes, 1991) consists of five scales with different demands: completing figures (detecting missing details in pictures), vocabulary test (explaining the meaning of terms), mosaic test (replicating patterns with small cubes), finding similarities between different terms, and mathematical reasoning (mental arithmetic).

\section{Statistical analyses}

As primary analysis, a repeated-measures ANOVA (ANOVA $\mathrm{RM}_{\mathrm{R}}$ ) of changes in adjusted MEP size after the PAS protocol was conducted with repeated factor "TIME" (baseline, post 0 , post 15 , post 30 , post 60 ) and between-subject factor "GENOTYPE" (BDNF Met carriers, BDNF Val/ Val carriers). To account for COMT and KIBRA carrier status, COMT and KIBRA genotype were entered into the model as additional betweensubject factors. Student's $t$ tests were run for post hoc comparisons if appropriate. Responders (grand average $>1$ ) and nonresponders to the PAS protocol were compared using $\chi^{2}$ tests.

Performance in the implicit learning tasks (AGL, SRTT) and in the neuropsychological test scores were compared using paired $t$ tests (BDNF) or univariate ANOVA (BDNF/COMT/KIBRA interactions).

Correlations between PAS-induced plasticity and implicit learning tasks were assessed using Pearson's or Spearman's correlations, as appropriate.

Statistical analyses were done using SPSS 19 (PASW, SPSS, IBM), and levels of significance were set to $p<0.05$.

\section{Results}

\section{Neurophysiological baseline measurements}

No differences were found between BDNF Val66Met genotype groups with regard to RMT, baseline MEPs, stimulation intensities or perceptual threshold (Table 4).

\section{PAS-induced plasticity}

One subject (BDNF Met carrier) had to be excluded from further analysis, due to extreme outliers in post-MEP sizes and grand average (data $>3$ SDs from the mean). The matched subject
(BDNF Val/Val carrier) was additionally excluded to counterbalance group sizes, leaving 30 subjects for group analyses.

For BDNF only, ANOVA $_{\mathrm{RM}}$ did not show a significant effect of BDNF genotype $\times$ TIME after PAS $\left(F_{(3,84)}=2.02, p=0.117\right)$; however, trend-wise larger MEPs at post 0 , post 15 , and post 60 in BDNF Val/Val carriers were noted compared with Met carriers (Fig. 3A).

When adding COMT Val158Met gene carrier status to the model, ANOVA $_{\mathrm{RM}}$ detected a significant interaction term $\operatorname{BDNF} \times \operatorname{COMT} \times \operatorname{TIME}\left(F_{(3,78)}=3.23, p=0.027\right)($ Fig. $3 B, C)$. Post hoc $t$ tests showed that in the group of homozygous COMT Met/Met carriers only, the BDNF Val/Val genotype was associated with a larger increase in MEP sizes at post 0 , compared with BDNF Met carriers $(p=0.01$; Fig. $3 B$ ). No differences were found within the group of COMT Val carriers ( $p>0.05$; Fig. $3 C$ ).

When adding KIBRA gene carrier status to the model, no significant effects were observed in combination with BDNF genotype, nor with both BDNF and COMT status ( $\mathrm{ANOVA}_{\mathrm{RM}}, p>$ 0.05 , data not shown).

In the two BDNF groups, distribution of responders (grand average, $>1$ ) and nonresponders were not significantly different $\left(\chi^{2}(1)=1.21, p>0.05\right)$. Likewise, no group differences were found for COMT and KIBRA interactions $(p>0.05)$.

\section{Implicit learning}

Artificial grammar learning

No significant differences between the two BDNF genotype groups $(n=32)$ for performance in the AGL task were found: both groups had comparable scores (percentage correct) in the classification task, $d^{\prime}$ values, and endorsement rates for grammatical and nongrammatical items (Table 5; Fig. $4 A$ ) (all $p>$ $0.05)$.

However, adding COMT Val158Met carrier status revealed distinct behavioral differences, namely a significant BDNF X COMT interaction term for endorsement rate of nongrammatical items (ANOVA, $F_{(28,1)}=5.97, p=0.021$ ) (Fig. $4 B, C$ ). Accordingly, BDNF Val/Val carriers that were also COMT Met/Met carriers showed higher task performance (as indicated by a lower tendency to perceive nongrammatical items as grammatical), compared with BDNF Met carriers of this group (Fig. $4 \mathrm{~B}$ ) (post hoc $t$ test, $p=0.07)$. In contrast, in the group of COMT Val carriers, BDNF Val/Val carriers exhibited lower task performance (as indicated by higher endorsement rates, thus a higher tendency to perceive nongrammatical items as grammatical), compared with BDNF Met carriers (Fig. $4 C$ ) (post hoc t test, $p=0.014$, n.s.).

No further interactions with COMT or KIBRA were found (ANOVA, all $p>0.05$ ).

\section{Serial reaction time task}

Both BDNF genotype groups showed similar motor learning performance in the SRTT $(n=32)$, with slightly higher values for BDNF Val/Val carriers (Fig. $5 A)\left(\right.$ ANOVA $\left._{\mathrm{RM}}, p>0.05\right)$. In COMT Met/Met carriers, an advantage of BDNF Val/Val carriers compared with BDNF Met carriers was noted (Fig. 5B), similar to results of AGL (see Artificial grammar learning), which failed to reach statistical significance though (ANOVA $p>0.05$ ). No differences were found in COMT-Val carriers.

No interaction effects were found with regard to KIBRA and COMT $\times$ KIBRA carrier status (all $p>0.05$ )

Notably, SRTT performance correlated significantly with PAS-induced plasticity (post 0; Spearman's correlation, $r=$ $0.52, p=0.003$ ) (Fig. 6). Performance in the AGL task did not correlate with PAS-induced plasticity (all $p>0.05)$. 

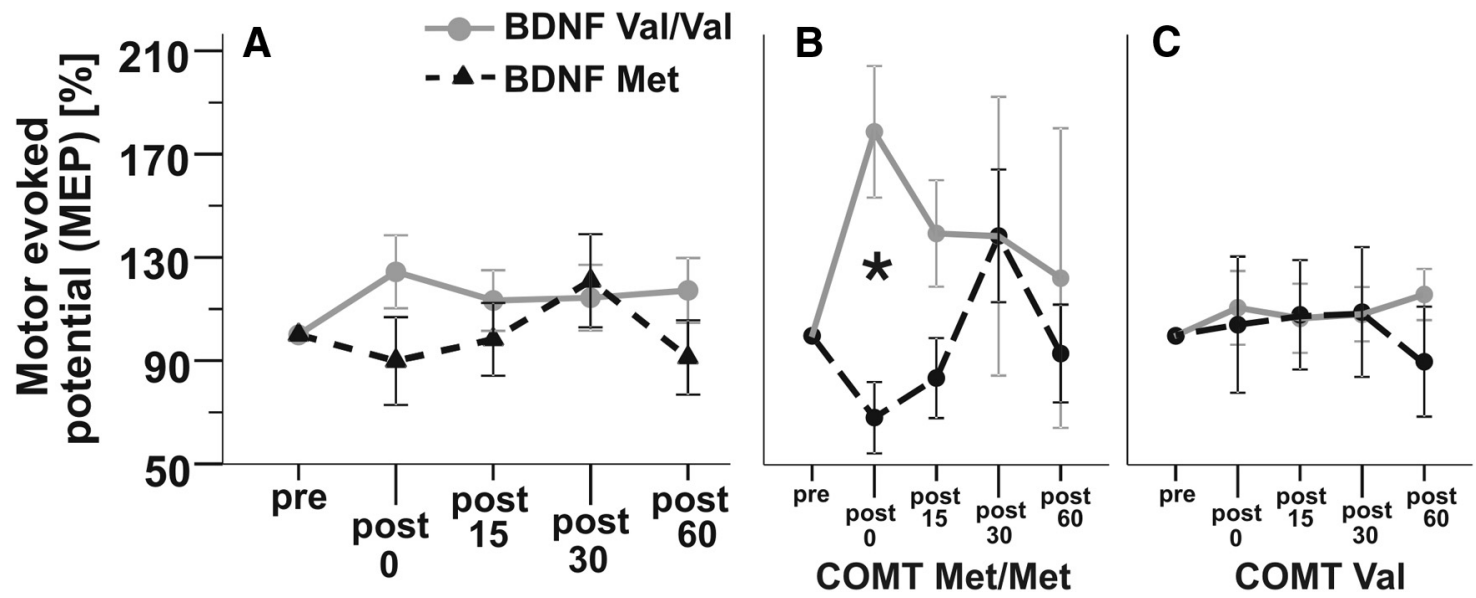

Figure 3. Effects of BDNF Val66Met alone and stratified for COMT Val158Met carrier status on cortical plasticity using $\mathrm{PAS}_{25} . A, B D N F$ Val/Val carriers (triangles, dashed line) exhibited larger MEP sizes than BDNF Met carriers (circles, gray line) after LTP induction relative to baseline (pre), most pronounced at post 0 ( ANOVA $_{\mathrm{RM}}, p=0.117$ ). $\boldsymbol{B}$, In COMT Met/Met carriers only, BDNF Val/Val genotype was significantly associated with an increase in MEP sizes pre vs post 0 compared with BDNF Met carriers, indicating higher PAS-induced plasticity in the combined BDNF Val/Val + COMT

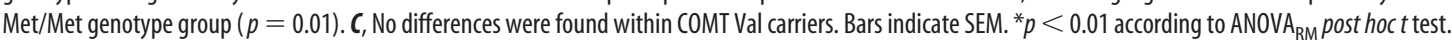

Table 5. Artificial grammar learning dependent on BDNF genotype

\begin{tabular}{lccc}
\hline & Met carriers & Val/Val carriers & $p^{*}$ \\
\hline Correct responses (\%) & $67.56 \pm 11.4$ & $66.44 \pm 9.1$ & 0.79 \\
$d^{\prime}$ & $1.04 \pm 0.8$ & $1 \pm 0.58$ & 0.89 \\
Endorsement rate, G items (\%) & $76.25 \pm 14.3$ & $75.63 \pm 14.2$ & 0.92 \\
Endorsement rate, NG items (\%) & $40.38 \pm 18.1$ & $43.38 \pm 15.2$ & 0.63 \\
\hline
\end{tabular}

No significant differences between genotype groups were found for correct responses, $\mathrm{d}^{\prime}$ or endorsement rate in the artificial grammar classification task. Data are given as mean \pm SD. *Paired $t$ test.

\section{Neuropsychological performance}

No significant differences were found between BDNF genotype groups on neuropsychological test performance (all $t$ values $<1.7$, all $p$ values $>0.1$ ) (Table 6).

No significant associations with COMT or KIBRA were found $(p>0.05)$, except a BDNF $\times$ COMT interaction for the subtest immediate learning for verbal paired associates $\left(F_{(1,28)}=8.21\right.$, $p=0.008$ ), showing higher scores in BDNF Val/Val carriers compared with BDNF Met carriers in the group of COMT Val carriers (post hoc $t$ test, $t=2.3, p=0.032$ ).

\section{Discussion}

We here present first-time evidence for a selective interaction effect of BDNF Val66Met and COMT Val158Met genotype on PAS-induced cortical plasticity in young females: while BDNF carrier status alone did not account for interindividual differences, plasticity was significantly higher in BDNF Val homozygotes if they were also COMT Met homozygotes, compared with BDNF Met carriers. These genotype-dependent effects were also found for grammar learning.

\section{BDNF Val66Met and brain stimulation}

In a key study by Kleim et al. (2006) (replicated by the same group, McHughen et al., 2010), increased MEP sizes and motor map representations after training of motor skills were found in young healthy $\mathrm{Val} / \mathrm{Val}$ carriers using TMS. Enhanced plasticity in $\mathrm{Val} / \mathrm{Val}$ carriers has been also observed using other brain stimulation protocols, i.e., TBS (Cheeran et al., 2008; Antal et al., 2010) and pharyngeal excitatory stimulation (Jayasekeran et al., 2011). An "advantage" of the BDNF Val allele with regard to plasticity has been explained by higher activity-dependent secretion of BDNF in Val carriers (Egan et al., 2003), as BDNF is implicated in
LTP (Poo, 2001). However, opposite effects were also reported, i.e., increased stimulation-induced plasticity in Met carriers using tDCS (Antal et al., 2010) and in response to inhibitory $1 \mathrm{~Hz}$ repetitive (r) TMS (Jayasekeran et al., 2011). Notably, recent studies indicated that an overexpression of BDNF may negatively affect plasticity and learning, due to hyperactivation of inhibitory pathways (for review, see Cunha et al., 2010). In the present study, we did not find significant effects on cortical plasticity induction using the PAS protocol for BDNF genotype alone. This finding is in line with previous studies reporting null findings (quadripulse stimulation; Nakamura et al., 2011) or trends only (PAS; Cheeran et al., 2008) if looking at BDNF genotype and measures of cortical plasticity.

The divergent results might be in part explained by methodological differences in stimulation techniques. For example, as the BDNF Val66Met polymorphism influences secretion of BDNF but does not influence its function (Egan et al., 2003), a longer duration of stimulation may be able to induce sufficient secretion of BDNF even in BDNF Met allele carriers. Thus, a stimulation protocol of $30 \mathrm{~min}$, as used in our own study and in that of Nakamura et al. (2011), might have diminished potential genotypedependent differences, in contrast to shorter protocols of $190 \mathrm{~s}$ or 13 min. Other intrinsic differences in protocols used to induce cortical plasticity in humans, e.g., stimulation mode or pulse frequency (Ziemann, 2004), may additionally contribute to differential findings between studies. We chose the PAS protocol (Stefan et al., 2000) for the present study as a frequently used and sensitive tool to investigate cortical plasticity and its relation to age and cognitive function in healthy subjects and neurological patients (Morgante et al., 2006; Weise et al., 2006; Frantseva et al., 2008; Muller-Dahlhaus et al., 2008; Castel-Lacanal et al., 2009; Fathi et al., 2010; Zeller et al., 2010; List et al., 2011, 2012).

Interaction effects of BDNF Val66Met and COMT Val158Met Additional possible confounders in this context are interactions of BDNF Val66Met with other SNPs that are suggested to modulate LTP, such as COMT and KIBRA. For example, the supposed BDNF genotype-dependent differences in BDNF concentrations and thus plasticity induction may be masked or evened out by COMT Val158Met-dependent differences in dopaminergic transmission, as dopamine is also known to affect LTP and neuronal plasticity (Wolf et al., 2003; Li et al., 2010). Indeed, after stratifying 
for COMT Val158Met genotype, we observed significantly increased PAS-induced cortical plasticity in BDNF Val homozygotes if subjects were also COMT Met homozygotes, compared with BDNF Met carriers. The COMT Met allele has been linked to less enzymatic activity of COMT and thus higher prefrontal dopamine (Lindenberger et al., 2008), yet whether COMT Val158Met carrier status influences human cortical plasticity has not been assessed so far. Several studies reported better executive function and memory performance in COMT Met carriers; however, others could not confirm these results (for review, see Witte and Flöel, 2011). Our findings for cortical plasticity indicated that the $\mathrm{BDNF} \mathrm{Val} / \mathrm{Val}$ genotype is beneficial only in combination with the low-activity COMT Met allele. Notably, group differences were most prominent immediately after PAS, yet diminished at 15, 30, and 60 min of followup. Bearing in mind the duration of the stimulation protocol (i.e., $30 \mathrm{~min}$ in the present study, see BDNF Val66Met and brain stimulation, above), the BDNF by COMT interaction found here might have affected immediate LTP-like plasticity only (for a detailed discussion, see also Stefan et al., 2000). A similar time course was also observed by Cheeran et al. (2008) for rTMSinduced effects, who found a significant BDNF Val66Met group difference immediately after a 15 min rTMS protocol, but not $10 \mathrm{~min}$ later. However, other stimulation protocols yielded longer-lasting group differences for BDNF Val66Met [e.g., following iTBS/cTBS (Cheeran et al., 2008) and tDCS (Antal et al., 2010)].

Immediate LTP affects the probability of transmitter release and has been suggested to be crucially important in "everyday memory” (Kandel, 2000; Wang et al., 2010). Thus, higher LTP-like plasticity directly after PAS should translate into behavioral advantages. Accordingly, the BDNF by COMT genotype group with increased LTP showed higher immediate learning success in the grammar task, and, although not reaching statistical significance, in the motor learning task. Moreover, LTP induction directly after PAS correlated with motor learning performance (Fig. 6). This is in line with previous studies that demonstrated higher LTP-like plasticity, induced with a 30 or 15 min PAS protocol, to be correlated with better cognitive functions in patients with cerebral white matter lesions (List et al., 2011) and better motor functions in patients with multiple sclerosis (Zeller et al., 2010). These findings, together with studies on LTP and learning in animal experiments (deToledo-Morrell et al., 1988; Tsien et al., 1996; Rosenzweig and Barnes, 2003), support the hypothesis that higher LTP, or LTP-like plasticity, correlates with better learning success.

One possible explanation for the genotype interaction effect observed in the current study might be that the positive effects of the BDNF-Val and COMT-Met alleles on BDNF concentrations and dopamine levels are small; therefore, subtle differences could
BDNF Val/Val

A BDNF Met

B

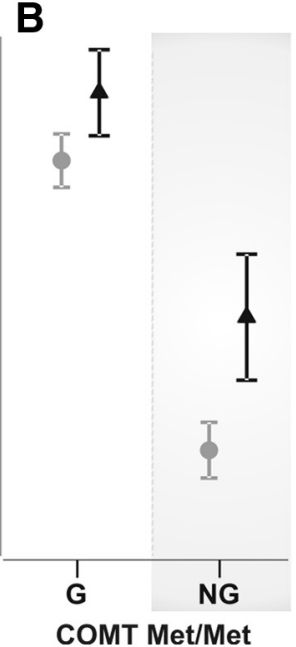

C

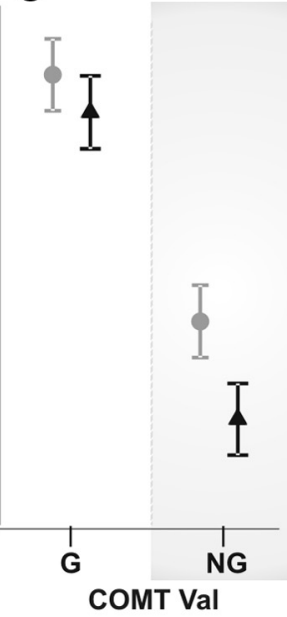

COMT Met/Met
COMT Val

Figure 4. Effects of BDNF Val66Met genotype alone and stratified for COMT Val158Met carrier status on artificial grammar learning. $A$, Endorsement rate, i.e., the percentage of items perceived as grammatical, was comparable between BDNF genotype groups for $G$ and NG boxes), showing that in COMT Met/Met carriers (B), BDNF Val/Nal carriers (gray circles) performed better than BDNF Met carriers (black

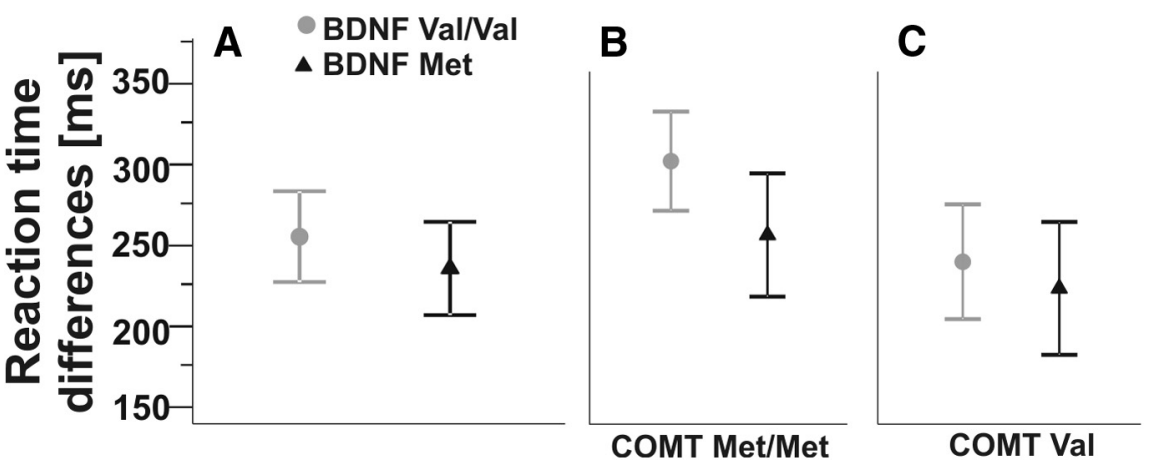

Figure 5. Effects of BDNF Val66Met genotype alone and stratified for COMT Val158Met carrier status on motor learning performance. $A$, Considering BDNF genotype alone, both groups exhibited similar motor learning, i.e., mean reaction time differences between sequential and random stimuli of the SRTT. B, BDNF Val/Nal carriers (gray circles) that were also COMT Met/Met carriers showed a trend for better performance compared with BDNF Met carriers (black triangles; ANOVA, n.s.). C, No differences were found in COMT Val carriers.

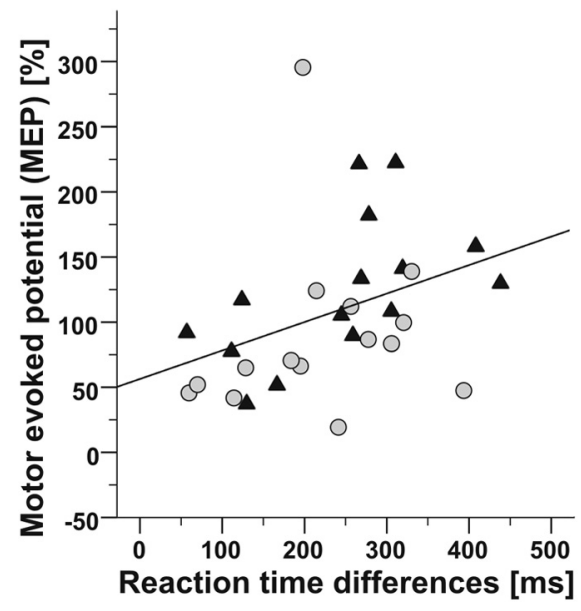

Figure 6. Correlation of motor learning performance and PAS-induced cortical plasticity. Mean reaction time differences between sequential and random stimuli (reaction time differences, $x$-axis) correlated significantly with the relative motor evoked potential (MEP, $y$-axis) at post 0 following the $\mathrm{PAS}_{25}$ protocol $(r=0.52, p=0.003)$. Gray circles, BDNF Met carriers; black triangles, BDNF Val $/ \mathrm{Nal}$ carriers. 
Table 6. Neuropsychological performance dependent on BDNF genotype

\begin{tabular}{|c|c|c|c|c|}
\hline Domain & Subtest & Met carriers & Val/Val carriers & $p^{*}$ \\
\hline \multirow[t]{6}{*}{ Learning and episodic memory } & AVLT, sum of trials 1-5 (learning) [words] & $63.69 \pm 5.9$ & $63.94 \pm 6.3$ & 0.87 \\
\hline & AVLT, trial 6 (recall after distraction) [words] & $13.69 \pm 1.8$ & $13.69 \pm 1.4$ & 1 \\
\hline & AVLT, trial 7 (delayed) [words] & $14.00 \pm 1.2$ & $13.63 \pm 1.3$ & 0.23 \\
\hline & AVLT, recognition trial (delayed) [words] & $14.63 \pm 0.5$ & $14.13 \pm 1.2$ & 0.11 \\
\hline & WMS-R, verbal paired associates [pairs] & $22.63 \pm 1.4$ & $22.94 \pm 1.6$ & 0.72 \\
\hline & WMS-R, visual paired associates [pairs] & $17.25 \pm 1.5$ & $17.13 \pm 0.9$ & 0.76 \\
\hline \multirow[t]{2}{*}{ Visuo-spatial construction and memory } & RCFT, copy [correct items] & $34.94 \pm 1.2$ & $34.94 \pm 1.1$ & 1 \\
\hline & RCFT, delayed copy [correct items] & $23.72 \pm 4.8$ & $25.41 \pm 5.1$ & 0.31 \\
\hline \multirow[t]{4}{*}{ Attention and working memory } & TMT, Part A $[\mathrm{sec}]$ & $20.92 \pm 5.2$ & $19.94 \pm 8.4$ & 0.7 \\
\hline & TMT, Part B [sec] & $49.18 \pm 16.3$ & $47.94 \pm 13.9$ & 0.81 \\
\hline & WMS-R, digit span [digits] & $18.00 \pm 2.3$ & $16.31 \pm 3.3$ & 0.17 \\
\hline & WMS-R, block span [blocks] & $17.75 \pm 2.1$ & $17.56 \pm 1.4$ & 0.33 \\
\hline \multirow[t]{4}{*}{ Verbal fluency } & RWT, lexical [words] & $26.50 \pm 5.3$ & $25.56 \pm 7.9$ & 0.68 \\
\hline & RWT, lexical category change [words] & $23.44 \pm 5.7$ & $22.50 \pm 5.8$ & 0.58 \\
\hline & RWT semantic [words] & $33.81 \pm 8.4$ & $38.19 \pm 6.0$ & 0.13 \\
\hline & RWT semantic, category change [words] & $21.19 \pm 3.5$ & $23.44 \pm 4.4$ & 0.14 \\
\hline General intellectual abilities & HAWIE-R $[\%]$ & $106.9 \pm 11.4$ & $105.6 \pm 12.8$ & 0.86 \\
\hline
\end{tabular}

Values are given as mean $\pm S D$. ${ }^{*}$ Values for paired $t$ test. No significant differences were found. For abbreviations, please see footnotes to Table 3.

only be seen when pooling the two genotypes to a "best of" combination. However, a genuine interaction effect may also be present and has been reported by previous studies for BDNF and COMT (Nagel et al., 2008). This is further supported by the fact that the high-activity BDNF Val homozygotes did not uniformly show better performance; rather, if paired with the "disadvantageous" lower dopamine COMT Val carriers, they showed significantly lower performance in grammar learning and immediate learning of verbal paired associates, compared with BDNF Met carriers of this group. Here, it may be hypothesized that reduced dopaminergic transmission in combination with high levels of BDNF would provoke rather adverse actions with regard to plasticity, e.g., hyperactivation of inhibitory pathways (Cunha et al., 2010). However, the exact underlying mechanisms remain elusive and have to be addressed in future experimental studies, e.g., using advanced molecular-genetic tools to explicitly study the effects of high BDNF concentration and low dopamine levels on neuronal activity. Notably, higher activity of COMT might not always lead to behavioral disadvantages, for example, regarding emotional tasks with higher demands of flexible versus stable neuronal processing within prefronto-limbic circuits (for a detailed discussion, see Witte and Flöel, 2011).

A slightly different interaction effect between BDNF and COMT has been reported for executive functions in older adults (Nagel et al., 2008). Yet in young adults in the Nagel et al. (2008) study, working memory performance for the BDNF by COMT interaction showed a similar pattern compared with that of our subjects in the grammar learning task (compare Nagel et al., 2008, their Fig. $2 D$, nonsignificant results). In sum, the impact of BDNF and COMT genotype on cortical plasticity and learning ability appears to be highly complex and remains to be fully elucidated, yet interactions between these two SNPs explained significantly more variance than considering BDNF genotype alone (this study; Nagel et al., 2008).

\section{Other learning-relevant SNPs}

For rs17070145 in the KIBRA gene, behavioral results have been mixed, ranging from beneficial effects of the T-allele (Papassotiropoulos et al., 2006; Almeida et al., 2008; Schaper et al., 2008; Vassos et al., 2010; Yasuda et al., 2010; Kauppi et al., 2011), to null findings (Need et al., 2008), to even poorer performance in T-allele carriers (Nacmias et al., 2008; Wersching et al., 2011). In line with Need et al. (2008), we did not find significant effects for KIBRA genotype on PAS-induced cortical plasticity, implicit learning, or neuropsychological performance. As previous studies with positive results included sample sizes of $>60$ subjects, the proposed effects of KIBRA genotype might be too small to yield significant results in our sample, especially with regard to triple interactions including BDNF and COMT genotype. Upcoming studies including larger sample sizes have to address this issue.

\section{Strength and limitations}

One limitation is the sample size of $n=32$, which might impact the statistical power to detect small genotype-dependent effects (see also Other learning-relevant SNPs, above). However, we used a sensitive measure, PAS-induced cortical plasticity, as primary outcome, and focused on one polymorphism, BDNF. For BDNF, subjects were carefully pairwise matched for age and education, and sample size was similar to previous studies using cortical plasticity measurements (Kleim et al., 2006; Cheeran et al., 2008; Antal et al., 2010; Nakamura et al., 2011). Still, no significant effect of BDNF alone on cortical plasticity or learning ability was detected. Moreover, as gender is a crucial factor that might interact with BDNF, COMT, and KIBRA (Wersching et al., 2011; Witte and Flöel, 2011), we included females only to fully avoid gender effects. Second, including only females entails that results cannot be directly transferred to males. Third, ceiling effects in our sample of healthy young subjects with regard to neuropsychological test scores may mask genotype-driven effects of BDNF, COMT, and KIBRA for these outcome parameters. Note that participants did not reach ceiling levels for cortical plasticity or implicit learning.

\section{Conclusion}

Our findings for the first time demonstrate an interaction of BDNF Val66Met and COMT Val158Met genotype on human cortical plasticity, explaining more interindividual variance than BDNF genotype alone. Moreover, they show that genotyperelated differences in neurophysiology also translate into behavioral differences. As a consequence, upcoming studies should account for possible interaction effects between different learning-relevant SNPs, to further clarify the ambiguous effects of single genotypes in the past. In sum, our findings might contribute to a better understanding of the mechanisms underlying 
interindividual differences in cognition, which might help to improve preventive and therapeutic strategies against neurological or psychiatric diseases (Mayor, 2007).

\section{References}

Almeida OP, Schwab SG, Lautenschlager NT, Morar B, Greenop KR, Flicker L, Wildenauer D (2008) KIBRA genetic polymorphism influences episodic memory in later life, but does not increase the risk of mild cognitive impairment. J Cell Mol Med 12:1672-1676.

Antal A, Chaieb L, Moliadze V, Monte-Silva K, Poreisz C, Thirugnanasambandam N, Nitsche MA, Shoukier M, Ludwig H, Paulus W (2010) Brain-derived neurotrophic factor (BDNF) gene polymorphisms shape cortical plasticity in humans. Brain Stimul 3:230-237.

Aschenbrenner A, Tucha O, Lange K (2000) RWT Regensburger WortflüssigkeitsTest. Handanweisung. Göttingen, Germany: Hogrefe Verlag.

Castel-Lacanal E, Marque P, Tardy J, de Boissezon X, Guiraud V, Chollet F, Loubinoux I, Moreau MS (2009) Induction of cortical plastic changes in wrist muscles by paired associative stimulation in the recovery phase of stroke patients. Neurorehabil Neural Repair 23:366-372.

Cheeran BJ, Ritter C, Rothwell JC, Siebner HR (2009) Mapping genetic influences on the corticospinal motor system in humans. Neuroscience 164:156-163.

Cheeran B, Talelli P, Mori F, Koch G, Suppa A, Edwards M, Houlden H, Bhatia K, Greenwood R, Rothwell JC (2008) A common polymorphism in the brain derived neurotrophic factor gene (BDNF) modulates human cortical plasticity and the response to rTMS. J Physiol 586:5717-5725.

Chen ZY, Jing D, Bath KG, Ieraci A, Khan T, Siao CJ, Herrera DG, Toth M, Yang C, McEwen BS, Hempstead BL, Lee FS (2006) Genetic variant BDNF (Val66Met) polymorphism alters anxiety-related behavior. Science 314:140-143.

Cleeremans A, McClelland JL (1991) Learning the structure of event sequences. J Exp Psychol Gen 120:235-253.

Cunha C, Brambilla R, Thomas KL (2010) A simple role for BDNF in learning and memory? Front Mol Neurosci 3:1.

deToledo-Morrell L, Geinisman Y, Morrell F (1988) Age-dependent alterations in hippocampal synaptic plasticity: relation to memory disorders. Neurobiol Aging 9:581-590.

de Vries MH, Barth AC, Maiworm S, Knecht S, Zwitserlood P, Flöel A (2010) Electrical stimulation of Broca's area enhances implicit learning of an artificial grammar. J Cogn Neurosci 22:2427-2436.

Egan MF, Goldberg TE, Kolachana BS, Callicott JH, Mazzanti CM, Straub RE, Goldman D, Weinberger DR (2001) Effect of COMT Val108/158 Met genotype on frontal lobe function and risk for schizophrenia. Proc Natl Acad Sci U S A 98:6917-6922.

Egan MF, Kojima M, Callicott JH, Goldberg TE, Kolachana BS, Bertolino A, Zaitsev E, Gold B, Goldman D, Dean M, Lu B, Weinberger DR (2003) The BDNF val66met polymorphism affects activity-dependent secretion of BDNF and human memory and hippocampal function. Cell 112:257-269.

Elwood RW (1991) The Wechsler Memory Scale-Revised: psychometric characteristics and clinical application. Neuropsychol Rev 2:179-201.

Erickson KI, Kim JS, Suever BL, Voss MW, Francis BM, Kramer AF (2008) Genetic contributions to age-related decline in executive function: a 10year longitudinal study of COMT and BDNF polymorphisms. Front Hum Neurosci 2:11.

Fathi D, Ueki Y, Mima T, Koganemaru S, Nagamine T, Tawfik A, Fukuyama H (2010) Effects of aging on the human motor cortical plasticity studied by paired associative stimulation. Clin Neurophysiol 121:90-93.

Flöel A, de Vries MH, Scholz J, Breitenstein C, Johansen-Berg H (2009) White matter integrity in the vicinity of Broca's area predicts grammar learning success. Neuroimage 47:1974-1981.

Forkstam C, Hagoort P, Fernandez G, Ingvar M, Petersson KM (2006) Neural correlates of artificial syntactic structure classification. Neuroimage 32:956-967.

Frantseva MV, Fitzgerald PB, Chen R, Möller B, Daigle M, Daskalakis ZJ (2008) Evidence for impaired long-term potentiation in schizophrenia and its relationship to motor skill learning. Cereb Cortex 18:990-996.

Helmstaedter C, Kurthen M (2001) Memory and epilepsy: characteristics, course, and influence of drugs and surgery. Curr Opin Neurol $14: 211-216$

Hochhaus L (1972) A table for the calculation of d' and Beta. Psychol Bull 77:375-376.

Jayasekeran V, Pendleton N, Holland G, Payton A, Jefferson S, Michou E,
Vasant D, Ollier B, Horan M, Rothwell J, Hamdy S (2011) Val66Met in brain-derived neurotrophic factor affects stimulus-induced plasticity in the human pharyngeal motor cortex. Gastroenterology 141:827-836.e1-3.

Jiménez L, Mendez C, Cleeremans A (1996) Comparing direct and indirect measures of sequence learning. J Exp Psychol Learn Mem Cogn 22:948-969.

Johannsen S, Duning K, Pavenstädt H, Kremerskothen J, Boeckers TM (2008) Temporal-spatial expression and novel biochemical properties of the memory-related protein KIBRA. Neuroscience 155:1165-1173.

Kandel E (2000) Cellular mechanisms of learning and the biological basis of individuality. In: Principles of neural science, Ed 4 (Kandel E, Schwartz JH, Jessel TM, eds), pp 1248-1279. New York: McGraw-Hill.

Kauppi K, Nilsson LG, Adolfsson R, Eriksson E, Nyberg L (2011) KIBRA polymorphism is related to enhanced memory and elevated hippocampal processing. J Neurosci 31:14218-14222.

Kleim JA, Chan S, Pringle E, Schallert K, Procaccio V, Jimenez R, Cramer SC (2006) BDNF val66met polymorphism is associated with modified experience-dependent plasticity in human motor cortex. Nat Neurosci 9:735-737.

Kurten J, De Vries MH, Kowal K, Zwitserlood P, Floel A (2010) Age affects chunk-based, but not rule-based learning in artificial grammar acquisition. Neurobiol Aging. Advance online publication. Retrieved February 24, 2012. doi:org/10.1016/j.neurobiolaging.2010.10.008.

Li SC, Lindenberger U, Bäckman L (2010) Dopaminergic modulation of cognition across the life span. Neurosci Biobehav Rev 34:625-630.

Lieberman MD, Chang GY, Chiao J, Bookheimer SY, Knowlton BJ (2004) An event-related fMRI study of artificial grammar leraning in a balanced chunk strength design. J Cogn Neurosci 16:427-438.

Lindenberger U, Nagel IE, Chicherio C, Li SC, Heekeren HR, Bäckman L (2008) Age-related decline in brain resources modulates genetic effects on cognitive functioning. Front Neurosci 2:234-244.

List J, Duning T, Meinzer M, Kürten J, Schirmacher A, Deppe M, Evers S, Young P, Flöel A (2011) Enhanced Rapid-Onset Cortical Plasticity in CADASIL as a Possible Mechanism of Preserved Cognition. Cereb Cortex 21:2774-2787.

List J, Duning T, Kürten J, Deppe M, Wilbers E, Flöel A (2012) Cortical plasticity is preserved in non-demented older individuals with severe ischemic small vessel disease. Hum Brain Mapp. Advance online publication. Retrieved February 24, 2012. doi:10.1002/hbm.22003.

Li Voti P, Conte A, Suppa A, Iezzi E, Bologna M, Aniello MS, Defazio G, Rothwell JC, Berardelli A (2011) Correlation between cortical plasticity, motor learning and BDNF genotype in healthy subjects. Exp Brain Res 212:91-99.

Mayor S (2007) Fitting the drug to the patient. BMJ 334:452-453.

McHughen SA, Rodriguez PF, Kleim JA, Kleim ED, Marchal Crespo L, Procaccio V, Cramer SC (2010) BDNF val66met polymorphism influences motor system function in the human brain. Cereb Cortex 20:1254-1262.

Meyers JE, Bayless JD, Meyers KR (1996) Rey complex figure: memory error patterns and functional abilities. Appl Neuropsychol 3:89-92.

Mizuno M, Yamada K, Olariu A, Nawa H, Nabeshima T (2000) Involvement of brain-derived neurotrophic factor in spatial memory formation and maintenance in a radial arm maze test in rats. J Neurosci 20:7116-7121.

Morgante F, Espay AJ, Gunraj C, Lang AE, Chen R (2006) Motor cortex plasticity in Parkinson's disease and levodopa-induced dyskinesias. Brain 129:1059-1069.

Müller-Dahlhaus JF, Orekhov Y, Liu Y, Ziemann U (2008) Interindividual variability and age-dependency of motor cortical plasticity induced by paired associative stimulation. Exp Brain Res 187:467-475.

Nacmias B, Bessi V, Bagnoli S, Tedde A, Cellini E, Piccini C, Sorbi S, Bracco L (2008) KIBRA gene variants are associated with episodic memory performance in subjective memory complaints. Neurosci Lett 436:145-147.

Nagel IE, Chicherio C, Li SC, von Oertzen T, Sander T, Villringer A, Heekeren HR, Bäckman L, Lindenberger U (2008) Human aging magnifies genetic effects on executive functioning and working memory. Front Hum Neurosci 2:1.

Nakamura K, Enomoto H, Hanajima R, Hamada M, Shimizu E, Kawamura Y, Sasaki T, Matsuzawa D, Sutoh C, Shirota Y, Terao Y, Ugawa Y (2011) Quadri-pulse stimulation (QPS) induced LTP/LTD was not affected by Val66Met polymorphism in the brain-derived neurotrophic factor (BDNF) gene. Neurosci Lett 487:264-267.

Need AC, Attix DK, McEvoy JM, Cirulli ET, Linney KN, Wagoner AP, Gumbs 
CE, Giegling I, Möller HJ, Francks C, Muglia P, Roses A, Gibson G, Weale ME, Rujescu D, Goldstein DB (2008) Failure to replicate effect of Kibra on human memory in two large cohorts of European origin. Am J Med Genet B Neuropsychiatr Genet 147B:667-668.

Nissen MJ, Bullemer P (1987) Attentional requirements of learning: evidence from performance measures. Cogn Psychol 19:1-32.

Oldfield RC (1971) The assessment and analysis of handedness: the Edinburgh inventory. Neuropsychologia 9:97-113.

Papassotiropoulos A, Stephan DA, Huentelman MJ, Hoerndli FJ, Craig DW, Pearson JV, Huynh KD, Brunner F, Corneveaux J, Osborne D, Wollmer MA, Aerni A, Coluccia D, Hänggi J, Mondadori CR, Buchmann A, Reiman EM, Caselli RJ, Henke K, de Quervain DJ (2006) Common Kibra alleles are associated with human memory performance. Science 314:475-478.

Patterson SL, Abel T, Deuel TA, Martin KC, Rose JC, Kandel ER (1996) Recombinant BDNF rescues deficits in basal synaptic transmission and hippocampal LTP in BDNF knockout mice. Neuron 16:1137-1145.

Poo MM (2001) Neurotrophins as synaptic modulators. Nat Rev Neurosci 2:24-32.

Reber AS (1967) Implicit learning of artificial grammars. J Verbal Learn Verbal Behav 5:855-863.

Rosenzweig ES, Barnes CA (2003) Impact of aging on hippocampal function: plasticity, network dynamics, and cognition. Prog Neurobiol 69:143-179.

Rösser N, Heuschmann P, Wersching H, Breitenstein C, Knecht S, Flöel A (2008) Levodopa improves procedural motor learning in chronic stroke patients. Arch Phys Med Rehabil 89:1633-1641.

Schaper K, Kolsch H, Popp J, Wagner M, Jessen F (2008) KIBRA gene variants are associated with episodic memory in healthy elderly. Neurobiol Aging 29:1123-1125.

Sen S, Nesse RM, Stoltenberg SF, Li S, Gleiberman L, Chakravarti A, Weder $\mathrm{AB}$, Burmeister M (2003) A BDNF coding variant is associated with the NEO personality inventory domain neuroticism, a risk factor for depression. Neuropsychopharmacology 28:397-401.

Spreen O, Strauss E (1998) A compendium of neuropsychological tests, Ed 2. New York: Oxford UP.

Stefan K, Kunesch E, Cohen LG, Benecke R, Classen J (2000) Induction of plasticity in the human motor cortex by paired associative stimulation. Brain 123:572-584.

Tewes U (1991) Hamburg-Wechsler intelligence test for adults, Ed 2. Göttingen, Germany: Hans Huber.
Tsien JZ, Huerta PT, Tonegawa S (1996) The essential role of hippocampal CA1 NMDA receptor-dependent synaptic plasticity in spatial memory. Cell 87:1327-1338.

Vassos E, Bramon E, Picchioni M, Walshe M, Filbey FM, Kravariti E, McDonald C, Murray RM, Collier DA, Toulopoulou T (2010) Evidence of association of KIBRA genotype with episodic memory in families of psychotic patients and controls. J Psychiatr Res 44:795-798.

Wang SH, Redondo RL, Morris RG (2010) Relevance of synaptic tagging and capture to the persistence of long-term potentiation and everyday spatial memory. Proc Natl Acad Sci U S A 107:19537-19542.

Weise D, Schramm A, Stefan K, Wolters A, Reiners K, Naumann M, Classen J (2006) The two sides of associative plasticity in writer's cramp. Brain 129:2709-2721.

Wersching H, Guske K, Hasenkamp S, Hagedorn C, Schiwek S, Jansen S, Witte V, Wellmann J, Lohmann H, Duning K, Kremerskothen J, Knecht S, Brand E, Floel A (2011) Impact of common KIBRA allele on human cognitive functions. Neuropsychopharmacology 36:1296-1304.

Witte AV, Flöel A (2011) Effects of COMT polymorphisms on brain function and behavior in health and disease. Brain Res Bull. Advance online publication. Retrieved February 24, 2012. doi: org/10.1016/ j.brainresbull.2011.11.012.

Witte AV, Jansen S, Schirmacher A, Young P, Flöel A (2010) COMT Val158Met polymorphism modulates cognitive effects of dietary intervention. Front Aging Neurosci 2:146.

Wolf ME, Mangiavacchi S, Sun X (2003) Mechanisms by which dopamine receptors may influence synaptic plasticity. Ann N Y Acad Sci 1003:241-249.

Yasuda Y, Hashimoto R, Ohi K, Fukumoto M, Takamura H, Iike N, Yoshida T, Hayashi N, Takahashi H, Yamamori H, Morihara T, Tagami S, Okochi M, Tanaka T, Kudo T, Kamino K, Ishii R, Iwase M, Kazui H, Takeda M (2010) Association study of KIBRA gene with memory performance in a Japanese population. World J Biol Psychiatry 11:852-857.

Yoshihama Y, Hirai T, Ohtsuka T, Chida K (2009) KIBRA Co-localizes with protein kinase Mzeta (PKMzeta) in the mouse hippocampus. Biosci Biotechnol Biochem 73:147-151.

Zeller D, aufm Kampe K, Biller A, Stefan K, Gentner R, Schütz A, Bartsch A, Bendszus M, Toyka KV, Rieckmann P, Classen J (2010) Rapid-onset central motor plasticity in multiple sclerosis. Neurology 74:728-735.

Ziemann U (2004) TMS induced plasticity in human cortex. Rev Neurosci $15: 253-266$ 